\title{
Increasing Black Start Capacity by Fast Cut Back Function of Thermal Power Plants
}

\author{
En Lu, ${ }^{\mathrm{a}, \mathrm{c}}$, Zhijun Qin ${ }^{\mathrm{b} *}$, Mingbo Liu ${ }^{\mathrm{a}}$, Yunhe Hou ${ }^{\mathrm{b}, \mathrm{d}}$, Ning Wang ${ }^{\mathrm{c}}$ and Jinyu Wen ${ }^{\mathrm{d}}$ \\ ${ }^{a}$ School of Electric Power, South China University of Technology, China \\ ${ }^{b}$ Department of Electrical and Electronic Engineering, The University of Hong Kong, Hong Kong, China \\ ${ }^{c}$ Guangdong Power Grid Dispatching and Communication Center, China Southern Power Grid, Guangzhou, China \\ ${ }^{d}$ Department of Electrical and Electronics Engineering, Huazhong University of Science and Technology, Wuhan, China
}

\begin{abstract}
Power system restoration is well recognized as one of the most important tasks for electric power grids. During the restoration process, preparation is the first stage, in which time is critical, and many non-blackstart units need to be cranked. The black start capacity plays a key role in preparation stage. This paper addresses thermal units with fast cut back (FCB) function as black start units. The model of FCB unit and algorithm for black start capacity assessment accounting for FCB unit is proposed. First, the general restoration process is briefly introduced. Second, the general restoration process is introduced by summarizing the industrial restoration practice. A piece of software entitled as "System Restoration Navigator" (SRN) is developed and used as a computational tool for restoration strategy assessment. Third, the model of FCB units and algorithm for assessment of FCB function in system restoration are proposed. Fourth, case studies show that FCB units increase black start capacity and reduce restoration time considerably. The proposed method has been successfully applied in Guangdong Power Company, China Southern Power Grid (CSG), where hydro power plants are limited and reliability requirement is critical.
\end{abstract}

Keywords: System restoration, black start capacity assessment, fast cut back

\section{Introduction}

Power system restoration is well recognized as one of the most important tasks for electric power grids. Following a power outage, dispatchers in the control center work with the field crews to re-establish the generation and transmission systems and then to pick up load and restore service. System reliability depends heavily on the efficiency of system restoration. The US Canada Report on the August 14, 2003 Blackout cites major power disturbances with the greatest impact, lasting between 15 and 50 hours [1],[2]. The data show that the impact of a blackout increases exponentially with the duration of its restoration.

A review of the literature and discussions with utility operators from different systems reveal that differences in "strategies" of restoration are closely related to differences in system characteristics. For a detailed restoration plan, to meet the particular requirements of an individual power system, it must be developed specifically. However, underlying these varying restoration strategies are commonalties in what might be termed "tactics" that are incorporated into the overall strategies. Generally, power systems share certain common characteristics and behave in a similar manner during the restoration process. It is therefore possible to establish a general procedure for system restoration[3], [4]. These stages may be termed preparation, system restoration, and load restoration [3], [5].

During the restoration process, preparation is the first stage, in which time is critical, and many urgent actions must be taken as quickly as possible. In this stage, usually, the post-disturbance system status is

\footnotetext{
* Manuscript received July 14, 2012; revised August 15, 2012.

Corresponding author. Tel.: +852 2857 8422; E-mail address: zjqin@eee.hku.hk.
} 
evaluated, the target system for restoration is defined, a strategy for rebuilding the transmission network is selected, the system may be partitioned into several subsystems, initial energy power sources are identified, steps are taken to crank non-blackstart (NBS) generating units, and paths are found to supply the critical loads. During this time-critical stage of restoration, it is desirable that actions be thoroughly planned for rapid implementation.

The initial source, or black start unit (BS), is an important issue for system restoration [3]. It is reported that restoration duration is reduced as the availability of initial sources of power decreases [4].

Initial sources are power sources that do not require off-site power to start. Usually, the initial sources that may be available during restoration is listed as follows[3], [4]:

- Hydro unit. In this category, run-of-the-river hydro and pumped-storage hydro can be designed for black start capability and have fast response characteristics. Usually, these two kinds of hydro units can be started in 5-10 minutes with a good probability of success.

- Diesel generating unit. Usually, a diesel generating unit is a small size unit. It is only employed for supplying the start up requirement of larger units, and cannot be used to pick up sizable loads or energize transmission lines. It has fast response characteristics.

- Gas turbines.

$>$ Aeroderivative gas turbine. It typically requires local battery power for cranking. It can be operated by remote operation and cam be used to pick up load quickly.

$>$ Lager gas turbine. Usually, to restart this type of unit, support from on-site diesel generating unit is necessary. The diesel generating unit is used to energize an auxiliary bus of the plant. The time for a gas turbine unit to be restarted is a function of the duration of that unit off-line.

- Tie-line with adjacent systems. If the tie-lines with adjacent systems are established, energy from the adjacent systems can be used as initial sources to crank non-blackstart units, pick up loads and energize transmission lines. Due to the system characteristics, tie-line with adjacent systems may be established quickly and reliably.

The thermal units are very difficult to implement black start after the blackout if there is no power injection from outside. Moreover, for power grids with thermal power plants in the highest flight, the system restoration will encounter difficulty due to lack of black start capacity. Fortunately, the fast cut back function (FCB) can keep the units running at rated speed with around 7-10\% normal load level. This function makes it possible for thermal power plants with FCB function to survive after a blackout or partial outage emergence. These FCB units serve as additional blackstart capacity, which will reduce the restoration time and improve the system reliability considerably. In China Southern Power Grid (CSG), a research project has been triggered to account for FCB function in system restoration.

Nevertheless, how the power grid benefits from the additional black start capacity introduced by FCB units remains an open question. This paper addresses the assessment of FCB units in accelerating the black start process. The remaining part of the paper is organized as follows. The general process of system restoration and the concept of Generic Restoration Milestones (GRMs) are introduced in section 2, which provides the theoretical basis for assessment of FCB function. The FCB function and specific configuration of one FCB unit in CSG are introduced in section 3. The algorithm and computational tool for assessment of FCB function is given in section 4. A case study with 7 generating units and 46 buses is given in section 5. Finally the conclusion is drawn in section 6 .

\section{Black start capacity for System Restoration}

\subsection{General process of system restoration}

System restoration is a gradual and iterative process with personnel safety and system stability as the primary considerations. When a disturbance occurs, a priority of system operation is to maintain the system integrity and stability. When the system is in an outage state, the objective is to restore services to customers as quickly as possible while safety and other constraints are satisfied. Therefore, the priorities of system operation under normal, emergency and restorative states are significantly different.

Following a major disturbance, remedial actions may be needed to steer the system to secure operating 
state. To meet this objective, the status of system, including system topology, availability of various components, need to be monitored and assessed first. Based on the system state, control actions will be determined. In case the disturbance leads to a power outage, the system restoration procedure will be invoked.

System restoration involves assessment of the system status, optimization of generation capabilities, restoration of the transmission and distribution networks, and load pickup. There are two fundamental issues in the development of decision support tools for system restoration. The first critical consideration of developing decision support tools for system restoration is the generality, i.e., tools should be portable from a system to another with minimal customization. A review of the literature and discussions with power system dispatchers from different systems reveal that differences in "strategies" of restoration are closely related to differences in system characteristics. As a result, it is a challenging task to develop a tool to support power system restoration efforts for a wide range of systems. For the same reason, a system restoration plan or guidelines are established off line for each individual power grid. However, the general process of system restoration can be concluded by summarizing the industry experience.

\subsection{Concept of generic restoration milestones (GRMs)}

Supported by EPRI, a systematic power system restoration methodology entitled Generic Restoration Milestones (GRMs) [5] are successfully developed. The objective of this methodology is to propose a general methodology for establishment of system restoration strategies. To cover different scenarios of system operations, the methodology should be adaptive and portable. For the objective, after careful identification the processes system restoration for different power systems, it is believed that the general process of system restoration can be decoupled into several general targets, which is entitled Generic Restoration Milestones.

GRMs provide a general toolbox for system restoration strategy establishment. In the context of the system conditions, a specific restoration strategy can be established by a combination of GRMs. Different combinations of GRMs will lead different system restoration strategy. Following a partial or complete outage, after analyzing system conditions, the system operators or system restoration planners can select a series of Milestones for this toolbox, and establish a restoration planning by combining GRMs.

Generally, the following 6 GRMs, are needed:

- GRM1: Form Black_Start_Non_Black_Start Building Block

- GRM2: Establish Transmission Grid

- GRM3: Form Electrical Island

- GRM4: Synchronize Electrical Islands

- GRM5: Serve Load in Area

- GRM6: Connect with Neighboring System

To ensure system security during a restoration process, a set of sophisticated algorithms have been developed. The targets of system restoration at different stages of system restoration are provided by GRMs. To accomplish these GRMs, a series of detailed actions are necessary. For this purpose, the concept called Generic Restoration Actions (GRAs), which is proposed in [6], will be employed.

\subsection{Black start capacity for system restoration}

The black start capability in system restoration is defined as the sum of MW generation capabilities over all units in the power system minus the startup power requirements. Following a blackout or a partial outage, some thermal units may require cranking power from outside in order to start the production process within the plant. It is of vital importance that, during system restoration, the black start capability is adequate to crank NBS units, otherwise the process of system restoration will be extended or even fail.

To achieve a faster restoration, adequate black start capacity is useful to accelerate the restoration process. When new black start generating units are installed, the restoration process will change in generator startup sequence, energizing path to NBS units, load pick-up amount, etc. How the additional black start capability benefits the restoration process needs to be indentified and quantified. On one hand, 
the restoration time will be changed with various FCB unit placement schemes. It is natural to quantify the benefit of FCB function by restoration time. On the other hand, BS units may not crank all NBS units due to system topology and operational constraints. FCB units will change the restoration process by changing the initial state of restoration. Multiple black start units will provide new options for a successful restoration plan.

\section{Fast Cut Back Unit}

\subsection{Introduction to FCB unit}

FCB units are designed and constructed to have the function which enables the units to bring the given load instantaneously down to the house in a stabilized mode of operation immediately after a trip from the power grid [7]-[10]. This is done by rapidly cutting back the fuel, feed water and air in response to the turbine-generator output. By triggering this function, the units running are always ready to return on line upon synchronization of the grid. Thus, the FCB capability would be beneficial for the system restoration of power grids as standby BS units.

The implementation of FCB function involves series of complex control actions in distributed control system (DCS). FCB control logic mainly consists several blocks as: boiler control, feedwater control, fuel control, air control, steam pressure control, rotation speed control, and steam temperature control, etc. The supper-heater bypass valve opens to reduce steam pressure when the unit loses large proportion of load. Other control blocks action coordinately to maintain stable temperature and rotation speed.

\subsection{Experiment of FCB units in CSG}

Currently in CSG, several FCB units have been set up as additional black start units. For example, Zhuhai Power Plant No. 1 unit is produced by Japan's Mitsubishi Heavy Industries, Ltd. The model is TC4F-40-type reheat condensing steam; boiler is radiation type and reheat forced circulation furnace, steam flow under is BMCR conditions $2290 \mathrm{t} / \mathrm{h}$. The generator is produced by the U.S. Westinghouse company, rated power is $746 \mathrm{MW}$ and power factor is 0.9 .

An experiment to test FCB function of Zhuhai No. 1 unit was conducted on Feb. 8th 2012. The unit took $700 \mathrm{MW}$ load before FCB function was triggered. The breaker connecting step-up transformer and the transmission line was trip manually to trigger FCB function. The dynamic process of the unit adjustment system quickly stabilized the entire unit. Transition process lasted about $12 \mathrm{~s}$. Steam turbine soaring speed was $3156 \mathrm{r} / \mathrm{min}$, which was $105.2 \%$ of rated speed. It is confirmed that Zhuhai Power Plant No. 1 unit has the ability of island operation, which contributes to restore the power grid back to normal state within the possible shorten time.

\section{Fast Cut Back Unit as a Black Start Unit}

\subsection{Modeling FCB unit in black start}

In black start computation, characteristics of different generating units should be involved. Based on the physical constraints of generating units, besides the parameters shared by all generating units, such as capacity, ramping rate, and minimal output, other different parameters are needed for different types of generating units. For example, for fossil units, startup power requirement and critical maximal interval are necessary; for super-critical-once-through (SCOT) units, startup power requirement and critical minimum interval should be considered; for black start units, such as hydro units, startup power requirement and time from cranking to parallel are zero [5], [8]. A generic model of generating unit [6] may be illustrated as Fig. 1, and the parameters are listed in Table 1.

For FCB units, the units survive after a black out or a partial outage with only house load. The output of FCB unit is positive, which means the unit is running to take load. No ancillary energy is needed to crank there units. FCB units can sustain low load level for a period before synchronization to power grid. After synchronized to power grid, these units begin to take load in the grid gradually to the minimal 
technical output level. Then these FCB units can ramp faster than nominal ramping rate since the boiler is still heated. The model for FCB units is shown in Fig. 2.

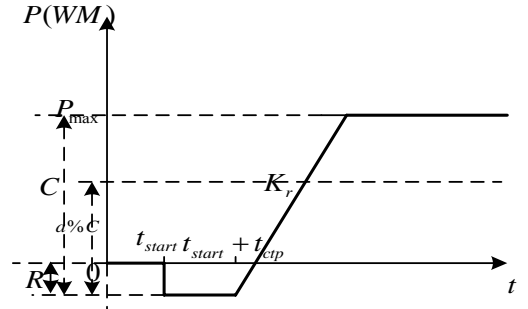

Fig. 1. Generic generating unit model.

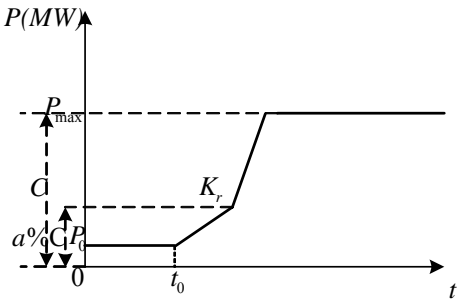

Fig. 2. FCB unit model.

Table 1. Parameters of generic generating unit

\begin{tabular}{llllll}
\hline Capacity & Startup requirement & Ramping Rate & Minimum output ratio & Crank to parallel & Begin to crank \\
\hline$C$ & $R$ & $K r$ & $\alpha \%$ & $t_{c t p}$ & $t_{\text {start }}$ \\
\hline
\end{tabular}

\subsection{Computational algorithm introduction}

The model to minimize the duration of black start can be expressed as:

$$
\min \sum_{s=1}^{N} \Delta t_{X_{s-1} \rightarrow X_{s}} \text { s.t. } H\left(X_{s}, U_{s}, Y_{s}\right)=0, G\left(U_{s}, Y_{s}\right) \leq 0, U_{s}=F\left(U_{s-1}, t\right)
$$

where $X_{s}$ is the system state of stage $s$ (set of energized buses, energized components' states), $Y_{s}$ is the system output of stage $s$ (bus voltages of energized grid), $U_{s}$ is the control of stage $s$ (the output of cranked generating units), $N$ is the number of NBS units, $H\left(X_{s}, U_{s}, Y_{s}\right)=0$ is the power flow equations, $G\left(U_{s}, Y_{s}\right) \leq 0$ stands for the feasible region, $U_{s}=F\left(U_{s-1}, t\right)$ stands for the output range of cranked generating units. $U_{s}=F\left(U_{s-1}, t\right)$ can be instantiated using the model illustrated in Fig. 5 and Fig. 6 .

Dur ing implementing black start, due to the stability of generating units and voltage of system, a part of load will be restored. It should be notified that at this stage, load restoration is only a means to the end. As a result, the implementation is decoupled into two sub-problems [6]:

- The primary problem: Finding the sequence to crank all of the non-blackstart units. The objective function is the minimal duration of the whole process. This is a classical multistage problem. Within the primary problem, restoration of each generating unit is modeled as a stage. At each stage, all of the available generating units will be crank. A tree with all of the possible restoration sequence is thus obtained. The dynamic programming will be employed to deal with this problem. Two essential algorithms will be involved in this problem:

- The secondary problem: Finding output of restarted generating units and picking up dispatchable load. This problem is formulated as a classical optimal power flow model.

A lot of complicated problems need to be solved in this bi-level optimization, including crank sequence of generating units, over-voltage on energizing transmission lines, frequency stability, transient stability, small signal stability, dynamic voltage stability, coordination with protective relay and communication, etc. Power system restoration decision support tools are needed for restoration planning and operation.

As for the restoration planning stage, a piece of software entitled "System Restoration Navigator" (SRN) is developed under the support of EPRI during the last two years. This software implements two essential functions, i.e., evaluation of system restoration strategy options, and establishment of a restoration strategy. The practical constrains have been involved into a restoration strategy evaluation and establishment step by step, and the flexible interactive mechanism can be employed to construct a reasonable restoration strategy associated with the users' expertise.

SRN is able to establish a sequence of generating units for system restoration plan following a complete or partial outage subject to steady-state constraints. This power system restoration plan can be 
established automatically and interactively. The program has the following functional features:

- The program establishes a system restoration plan following a complete or partial outage.

- Graphical user interface allows the user to enter or modify the input parameters, execution modules and view the outputs.

- Both source data and calculation results are displayed in tables or a one-line diagram.

- Users can set milestone and priority for each generating unit and critical loads in a table and a one-line diagram.

- Users can import/export characteristics of each generating unit, load, branch and transformer into *.char files.

- The user can save a system restoration plan in series of TXT files or image files step by step.

SRN implements the bi-level optimization algorithm mentioned above and is employed to assess the FCB function in system restoration.

\section{Case study}

In this section, a revised 46 buses power grid, which is part of a realistic US power grid, is used to assess FCB function in system restoration. This number of components is listed in Table 2. The parameters of generating units are listed in Table 3.

Table 2. Number of components

\begin{tabular}{cc}
\hline Type & Number \\
\hline Buses & 46 \\
Generators & 8 \\
AC lines & 41 \\
Transformers & 11 \\
Dispatchable loads & 23 \\
\hline
\end{tabular}

Table 3. Generator parameters

\begin{tabular}{cccccc}
\hline Bus & Type & Capacity(MW) & Start Requirement(MW) & Ramping rate(MW/hour) & Time to crank(hour) \\
\hline 5412 & NBS & 8 & 10 & 20 & 1 \\
5413 & NBS & 12 & 10 & 20 & 1 \\
6780 & BS & 36 & 0 & 36 & 0 \\
6781 & NBS & 172 & 10 & 28 & 2 \\
6782 & NBS & 205 & 20 & 34 & 2 \\
6783 & NBS & 216 & 10 & 108 & 2 \\
6784 & NBS & 150 & 10 & 150 & 0.75 \\
6785 & NBS & 150 & 10 & 150 & 0.75 \\
\hline
\end{tabular}

First, only one BS unit, i.e. unit on bus 6780 is assigned, as in Table 3. After computation in SRN, the time needed to crank all NBS units is 113.86 minutes.

Second, assuming one more generating unit is equipped with FCB function in different bus, restart the computation and assess the time again. When some units are assigned as with FCB function, their startup requirement and time to crank are set as 0 . The computational result is illustrated in Fig. 3, which shows the crank time is reduced to 81.81 minutes with FCB function on bus 6785 . In other words, adding one more FCB unit on bus 6785 leads to up to $28 \%$ acceleration in black start. From the data listed in Table 3, units on bus 6785 and bus 6784 have the fastest ramping rate $150 \mathrm{MW} / \mathrm{h}$, which contribute to the maximum acceleration in black start. It is also shown from Fig. 7 that appropriate install strategy of FCB units will provide more additional black start capacity for a faster and stable restoration process.

Third, we assume 2 units with FCB function on bus 5413, and bus 6785, respectively. There are totally 3 units with black start capacity. The computational result is that the black start time is 65.00 minutes, which is only $57 \%$ compared to with only on BS unit. It is shown that, if the FCB units are installed close to NBS units, the duration of black start is also reduced as a result of shorter energizing path. 


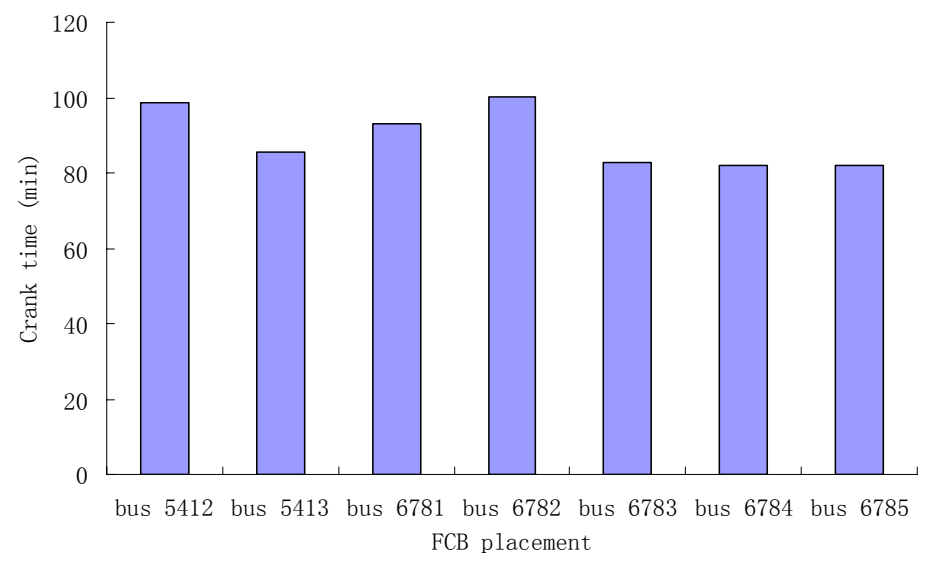

Fig. 3. Crank time of installing one more FCB unit in different bus

\section{Conclusion}

It is recognized that black start capacity plays a key role in system restoration. Under the condition that hydro units are limited, system restoration will encounter great difficulty. FCB function makes it possible that thermal units run stable with only house load and ready to connect to power grid when needed. Hence FCB units serve as black start units in CSG, especially in Guangdong province, which is the most important developed region in China.

This paper introduces FCB function in system restoration. The model of FCB unit and algorithm for assessment of FCB function in system restoration are proposed. Case studies show that FCB function can reduce the restoration time considerably.As a restoration planning tool, SRN is developed and has been employed in realistic power industry environment. It is efficient in assessment of restoration strategies.

\section{References}

[1] Adibi MM and Fink LH. Overcoming restoration challenges associated with major power system disturbances-restoration from cascading failures. IEEE Power and Energy Magazine, 2006; 4:68-77.

[2] Adibi MM and Martins N. Power system restoration dynamics issues, In: Proc. of IEEE Power and Energy Society General Meeting 2008:1-8.

[3] Adibi MM and Fink LH. Overcoming restoration challenges associated with major power system disturbances-restoration from cascading failures. IEEE Power and Energy Magazine, 2006; 4:68-77.

[4] Adibi MM and Martins N. Power system restoration dynamics issues. In: Proc. of IEEE Power and Energy Society General Meeting 2008:1-8.

[5] Fink LH, Liou KL, Liu CC. From generic restoration actions to specific restoration strategies. IEEE Trans. on Power Systems, 1995; 10(2): 745-752.

[6] Hou YH, Liu CC, Sun K, Zhang P, Liu SS, Mizumura D. Computation of milestones for decision support during system restoration. IEEE Trans. on Power Systems, 2011; 26(3):1399-1409.

[7] Kitada M, Masuya T, Tanaka H. Experimental house load operation at Tanagawa No.2 oil-fired power station. IEEE Trans. on Power Apparatus and Systems, 1981; PAS-100(6):2796-2803.

[8] De Mello FP, Westcott JC. Steam plant startup and control in system restoration. IEEE Trans. on Power Systems, 1994; 9(1):93-101.

[9] Kuchitsu M, Hrashima T, Fukushima K, Sato Y, Morio M. Fast cutback techniques for large-scale thermal power station, HItachi Review, 1978; 27(3):161-166.

[10] Huang W. Research and study of FCB test based on conventional configuration. In: Proc. of 2011 International Conference on Electonics, Commications and Control, 2011:4448-4450. 